\title{
Tailoring Noninvasive Ventilation Management in Non-ICU Settings and the Cardiac Surgery Context
}

Most respiratory therapists agree that noninvasive ventilation (NIV) has brought a revolution in respiratory failure. ${ }^{1}$ This rapidly expanding treatment strategy supports gas exchange and improves functional status of patients, shortens ICU and hospital stay, reduces mortality, and decreases costs. ${ }^{2}$ NIV is now the accepted standard of care for high-risk patients with exacerbation of COPD or acute cardiogenic pulmonary edema. ${ }^{1}$ The use of NIV has also increased in other acute respiratory failure etiologies, such as acute lung injury and ARDS, severe communityacquired pneumonia, chest trauma, and in immunocompromised patients. ${ }^{3}$ During the past decade, therapeutic applications of NIV have been reported in postoperative respiratory failure ${ }^{4,5}$ and in acute respiratory distress after extubation, as a first-line intervention, though with controversial effectiveness. ${ }^{6}$

Indisputably, the effectiveness of NIV commencement depends on the establishment of a successful institutional NIV program. Training in NIV is of utmost importance and must be thorough. Identifying appropriate patients and understanding the goals and the risks of NIV can be reached only with continuing education. Improved knowledge among clinicians increases its successful and efficient use. Other important factors include the available respiratory equipment and the acceptance of a proper protocol. ${ }^{2}$ Moreover, choosing the appropriate location for applying NIV can be a significant part of a flexible NIV program, although that may contain risks. Establishing an NIV program in a non-ICU setting can be safely performed, but the setting selection requires consideration of several factors: staff education and skills, the unit's monitoring capabilities, and identifying the more stable patients are the keys to success. ${ }^{7}$ Given the considerable pressure for ICU beds, this option is quite attractive and promising in many hospitals. An ideal place for NIV would seem to be the stepdown unit, which offers lower cost and a less distressful environment than the ICU. Additionally, the staff's NIV experience and the monitoring available in a step-down unit ensure the efficiency of NIV management. In some circumstances, and in the lack of an available high-dependence-unit bed, physicians are forced to initiate NIV on the general wards. However, both technical and personnel resources are required. In the absence of an experienced NIV team, and if the ward is not suitably monitored, there should be concern about patient safety and NIV failure. In this issue of Respiratory CARE, Khalid et al evaluate the outcomes of NIV delivered on general wards by a medical emergency team. According to their results, NIV can be safely initiated by specialized and educated personnel outside the ICU. ${ }^{8}$

See the Original Study on Page 186

NIV has already entered into new specialties as a treatment strategy, and according to the literature it has rapidly expanded over the last decade also in the field of cardiac surgery, as pulmonary dysfunction is one of the most frequent and serious post-cardiac surgery complications. ${ }^{9}$ Cardiopulmonary bypass, which takes over the function of the heart and lungs during surgery, involves risks from factors including blood contact with artificial material, administration of heparin-protamine, hypothermia, and lung ventilatory arrest, leading to the activation of pathways of systemic inflammatory response. ${ }^{10}$ Lung injury after cardiopulmonary bypass, also known as "post-bypass lung," 11 is associated with functional, physiologic, biochemical, and histological changes. ${ }^{10}$ Moreover, general anesthesia, sternotomy, breach of the pleura, hypothermia, and fluid overload are also of paramount importance in the development of postoperative pulmonary dysfunction. ${ }^{11}$ Of course, the influence on the cardiovascular system of positive pressure and volume delivered through the airways, which can be highly favorable on the impaired left heart and less favorable on the diseased right heart, should be considered when applying NIV in a cardio-surgical patient.

NIV has been evaluated in post-cardiac-surgery patients to prevent or treat postoperative acute respiratory failure. It has been increasingly used both in and outside the ICU, and benefits respiratory and cardiovascular function. ${ }^{12} \mathrm{Con}-$ sidering the limited availability of beds in the cardiac surgery ICU, the necessity — but also the opportunity - to apply NIV in step-down units and wards emerged. As a matter of fact, little is known about NIV use in cardiac surgical wards, due to a paucity of data. Usually a respiratory therapy service manages the treatment in conjunction with cardiac surgeons and ward nurses. A prudential approach is required, but the results could be very satis- 
factory for the surgeons, and above all for the patient. By shortening ICU stay and avoiding reintubation and ICU readmission, NIV may be very useful. Despite these positive results, in small retrospective single-center studies, a failure rate of $10-55 \%$ has been reported, which has to be estimated in more detail. ${ }^{13}$ Do we need larger randomized controlled trials, particularly in treating postoperative acute respiratory failure? Does safer and more effective use of NIV require the establishment of a successful NIV program in this area also? Who should receive NIV? When, where, and how? This new horizon of NIV application requires definite answers to such questions. Considering that optimal NIV management in this new field is unknown, reliable protocols and programs should be developed. Clearly indentifying NIV indications and contraindications in post-cardiac-surgery patients by a trained and experienced NIV team is needed. We hope that new clinical trials and ongoing discussion may clarify the preventive and treatment role of NIV in cardiac surgery. ${ }^{1}$

Charilaos-Panagiotis C Koutsogiannidis MD Olga G Ananiadou MD

Cardiothoracic Surgery Department General Hospital G Papanikolaou Thessaloniki, Greece

The authors have disclosed no conflicts of interest.

Correspondence: Charilaos-Panagiotis C Koutsogiannidis MD, Cardiothoracic Surgery Department, General Hospital G Papanikolaou, Exohi 57010, Thessaloniki Greece. E-mail: harisdoc76@yahoo.gr.

DOI: $10.4187 /$ respcare. 03035

\section{REFERENCES}

1. Kallet RH. Noninvasive ventilation in acute care: controversies and emerging concepts. Respir Care 2009;54(2):259-263.

2. Davies JD, Gentile MA. What does it take to have a successful noninvasive ventilation program? Respir Care 2009;54(1):53-61.

3. Keenan SP, Mehta S. Noninvasive ventilation for patients presenting with acute respiratory failure: the randomized controlled trials. Respir Care 2009;54(1):116-126.

4. Jaber S, Chanques G, Jung B. Postoperative noninvasive ventilation. Anesthesiology 2010;112(2):453-461.

5. Koutsogiannidis CP, Ampatzidou FC, Ananiadou OG, Karaiskos TE, Drossos GE. Noninvasive ventilation for post-pneumonectomy severe hypoxemia. Respir Care 2012;57(9):1514-1516.

6. Su CL, Chiang LL, Yang SH, Lin HI, Cheng KC, Huang YC, Wu CP. Preventive use of noninvasive ventilation after extubation: a prospective, multicenter randomized controlled trial. Respir Care 2012;57(2):204-210

7. Hill NS. Where should noninvasive ventilation be delivered? Respir Care 2009;54(1):62-70.

8. Khalid I, Sherbini N, Qushmaq I, Qabajah MR, Nisar A, Khalid TJ, Hamad WJ. Outcome of patients treated with noninvasive ventilation by Medical Emergency Team on the wards. Respir Care 2013 [ePub ahead of print]

9. Asimakopoulos G, Smith PL, Ratnatunga CP, Taylor KM. Lung injury and acute respiratory distress syndrome after cardiopulmonary bypass. Ann Thorac Surg 1999;68(3):1107-1115.

10. Ng CS, Wan S, Yim AP, Arifi AA. Pulmonary dysfunction after cardiac surgery. Chest 2002;121(4):1269-1277.

11. Carvalho EM, Gabriel EA, Salerno TA. Pulmonary protection during cardiac surgery: systematic literature review. Asian Cardiovasc Thorac Ann 2008;16(6):503-507.

12. Landoni G, Zangrillo A, Cabrini L. Noninvasive ventilation after cardiac and thoracic surgery in adult patients: a review. J Cardiothorac Vasc Anesth 2012;26(5):917-922.

13. Cabrini L, Plumari VP, Nobile L, Olper L, Pasin L, Bocchino S et al. Noninvasive ventilation in cardiac surgery: a concise review. HSR Proc Intensive Care Cardiovasc Anesth 2013 (in press). 